\title{
Constance des valeurs humanistes chez Montaigne et chez Shakespeare
}

\section{Robert Ellrodt}

\section{(2) OpenEdition Journals}

Édition électronique

URL : http://journals.openedition.org/shakespeare/153

DOI : 10.4000/shakespeare. 153

ISSN : 2271-6424

Éditeur

Société Française Shakespeare

Édition imprimée

Date de publication : 1 novembre 2004

Pagination : 95-116

ISBN : 2-9521475-0-7

Référence électronique

Robert Ellrodt, «Constance des valeurs humanistes chez Montaigne et chez Shakespeare », Actes des congrès de la Société française Shakespeare [En ligne], 21 | 2004, mis en ligne le 27 janvier 2007, consulté le 30 avril 2019. URL : http://journals.openedition.org/shakespeare/153 ; DOI : 10.4000/ shakespeare.153 


\section{Shakespeare et Montaigne vers un nouvel humanisme}

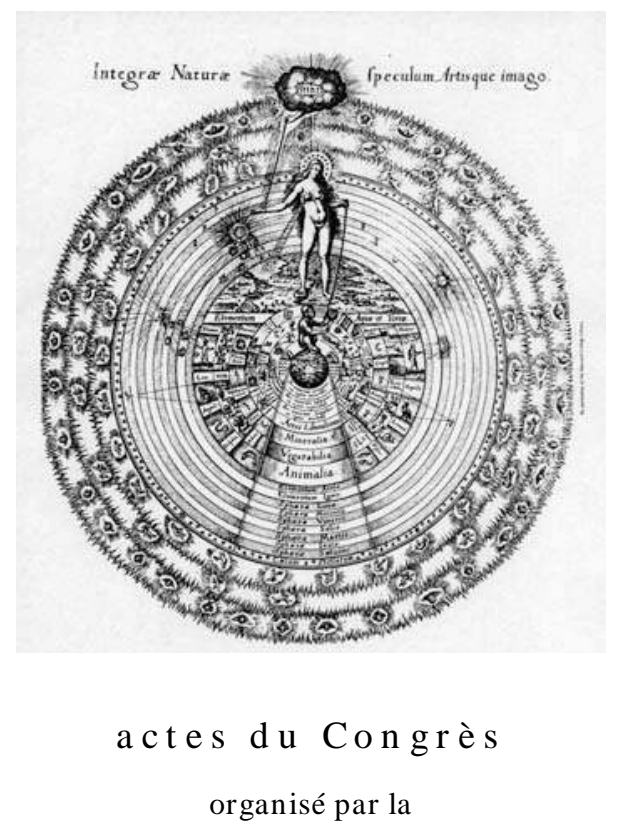

SOCIÉTÉ FR ANÇAISE SH AKESPEARE en collaboration avec la

S OC I É T É I N T E R N A T I O N A L E DES AM I D E M O N A I G N les 13,14 et 15 mars 2003

$$
\begin{gathered}
\text { textes réunis par } \\
\text { Pierre KAPITANIAK } \\
\text { sous la direction de } \\
\text { Jean-Marie MAGUIN }
\end{gathered}
$$




\section{COMITÉ SCIENTIFIQUE :}

Margaret Jones-Davis

Gisèle Venet

Jean-Marie Maguin

Yves Peyré

François Laroque

Pierre Kapitaniak

\section{COUVERTURE :}

Robert Fludd

Utriusque Cosmi Historia (1617-19)

planche 17

conception graphique et logo

Pierre Kapitaniak

\section{(C) 2003 Société Française Shakespeare}

Institut du Monde Anglophone

Université de Paris III - Sorbonne Nouvelle

http:// univ-montp3.fr/SFS/

5 rue de l'École de Médecine

75006 Paris

Diffusion :

AVL DIFFUSION

Parc Euromédecine

34198 MONTPELLIER CEDEX 5

ISBN 2-9521475-0-7

Tous droits de traduction, de reproduction et d'adaptation réservés pour tous les pays 


\title{
CONSTANCE DES VALEURS HUMANISTES CHEZ MONTAIGNE ET CHEZ SHAKESPEARE
}

\author{
Robert ELLRODT
}

\begin{abstract}
Un relativisme moral et culturel prévaudrait dans plusieurs pièces de Shakespeare à partir d'Hamlet, peut-être sous l'influence du scepticisme de Montaigne. Pourtant ils privilégient l'un et l'autre des valeurs " humanistes " qu'ils semblent considérer comme universelles plutôt que "relativement stables" dans le cadre d'une " période ». En reprenant et en nuançant les critères de Todorov pour une définition de l'humanisme, on peut tracer un parallèle entre l'essayiste et le dramaturge. Leur "compréhension de l'autre ", portée au plus haut point dans l'amitié, s'étend au commun des hommes, à l'étranger, au sauvage (sans illusion, même dans l'essai sur les Cannibales), et même à l'animal. Ils dénoncent « l'inhumanité de l'homme envers l'homme » et la folie des guerres civiles. La tragédie shakespearienne n'est pas un théâtre de la cruauté : l'appel à la compassion y est insistant. Ces valeurs morales sont assumées par un sujet, un « je ", qui, malgré la flexibilité du moi, est capable de se gouverner et de se former. L'essayiste se défie de la raison spéculative, mais il a une certaine confiance en son jugement, en sa "capacité de trier le vrai ". Vérité, réalité, essence sont inséparables ; Montaigne et Shakespeare sont parfois dits à tort " anti-essentialistes ", mais il est vrai qu'ils s'intéressent seulement à l'essence particulière d'un être singulier. Ils n'aspirent ni l'un ni l'autre à une transcendance verticale, sur le modèle des humanistes platonisants ou stoïciens, mais parviennent à se transcender vers l'autre dans l'amitié et dans la création littéraire.
\end{abstract}

Constant humanistic values in Montaigne and Shakespeare $A$ moral and cultural relativism is said to prevail in several plays of Shakespeare, from Hamlet onward, perhaps under the influence of Montaigne's scepticism. Yet both authors privilege humanistic values which they seem to consider as universal rather than "relatively stable" and confined to a "period". Adopting, yet modifying slightly, the criteria of Todorov for a definition of "humanism", one can point out parallel traits in the attitudes of the essayist and the works of the dramatist. Their "comprehension of the other", seen at its highest in friendship, can embrace the common man, the foreigner, the savage (without any illusion, even in the essay "Of Cannibals") and extends to, animals. They denounce man's inhumanity to man and the madness of civil wars. Shakespearian tragedy is not a "theatre of cruelty": it makes an insistent appeal to pity. These moral values are assumed by a "subject" capable of selfcontrol and deliberate self-fashioning despite the flexibility of the self. The essayist distrusts speculative reason, but places some confidence in his own judgment and his "capacity of sifting out the truth". Truth, reality, "essence" are inseparable. Montaigne and Shakespeare are sometimes wrongly said to be "anti-essentialist", but it is true that they are only interested in the particular essence of an individual being. Neither of them aspired to a vertical transcendence on the Platonic or Stoic pattern, but each of them succeeded in transcending himself in friendship and in the experience of literary creation.

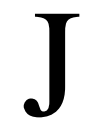

'ai traité deux fois déjà des rapports entre Shakespeare et Montaigne. Une étude a paru dans Shakespeare Survey en 1975. Une conférence, prononcée au World Shakespeare Congress de 2001, «Self-consistency in Montaigne and Shakespeare », sera publiée cette année même ${ }^{109}$. J'ai donc choisi une perspective différente qui me contraindra parfois à ne présenter que des idées générales, quitte à renvoyer aux travaux précédents.

109 Dans les Proceedings édités sous le titre Shakespeare and the Mediterranean, University of Delaware Press, 2003. 
Par ce titre ambigu je n'entends pas rappeler que Montaigne et Shakespeare ont fréquenté les auteurs classiques. Cela relève de l'évidence dans le cas de Montaigne et nous ne sommes plus au temps où l'on célébrait les native woodnotes wild de Shakespeare. De T. M. Baldwin à Robert Miola et Jonathan Bate, les traces nombreuses de la culture acquise dans l'excellente «grammar school» de Stratford ont été relevées dans son oeuvre ${ }^{110}$. Renvoyant à ces amples travaux, je m'arrête à un détail. Le bouffon de cour Touchstone dans As You Like $I t$, exilé dans la Forêt d'Ardennes parmi les chèvres, se compare à Ovide exilé «parmi les Goths » en jouant sur l'homophonie entre Goth et goat (III.iii.6). Se sentir de plain-pied avec les poètes et les personnages de l'Antiquité a permis au dramaturge de nous les rendre si présents et si proches. Ainsi s'explique aussi qu'il n'ait pas été rebuté dans sa lecture des Essais de Montaigne par la profusion de citations tirées des auteurs anciens. La culture littéraire de la Renaissance a été une culture de l'allusion aux textes classiques comme à l'Écriture sainte. En témoigne la prolifération des recueils de sentences et d'adages; les plus grands humanistes, comme Érasme, n'ont pas dédaigné d'en compiler et la sagesse de Shakespeare est souvent une sagesse proverbiale ${ }^{111}$.

S'il est évident que les lectures du poète élisabéthain furent moins étendues que celles de Montaigne, il n'en est pas moins remarquable qu'ils eurent en commun un vif intérêt pour Ovide dont Géralde Nakam a souligné l'influence sur les Essais ${ }^{112}$. Un même intérêt aussi pour les historiens, Tite Live et surtout Plutarque, y cherchant tous deux, plutôt que les actions publiques, « les devis que [Brutus] tenoit en sa tente à quelqu'un de ses privez amis, la veille d'une bataille» (II, 10, 394a $)^{113}$. Et une semblable réaction à l'égard de

\footnotetext{
110 T. M. Baldwin, William Shakspere's Small latine and Lesse Greek, 2 vol., Urbana, University of Illinois Press, 1944; R. Miola, Shakespeare's Reading, Oxford, O. U. P., 2000 ; J. Bate, Shakespeare and Ovid, Oxford, O. U. P., 1993. — Sur les caractéristiques originales de l'utilisation des classiques par Montaigne, voir R. Aulotte, "Montaigne et l'humanisme » dans Montaigne Penseur et philosophe, éd. C. Blum, Paris, Champion, 1990. ${ }^{111}$ Voir F. P. Wilson, The Proverbial Wisdom of Shakespeare, Londres, Modern Humanities Research Association, 1961.

112 Le dernier Montaigne, Paris, Champion, 2002, p. 3.

113 Édition utilisée: Euvres complètes, éd. Thibaudet-Rat, Paris, Gallimard, coll. «Pléiade », 1962.
} 
l'aspiration stoïcienne à la constance malgré les différences subtilement analysées par Geoffrey Miles ${ }^{114}$.

Tout ceci est connu et admis. Présenter Montaigne et Shakespeare comme des humanistes en un sens philosophique n'a rien de nouveau non plus. Mais les études parallèles récentes, de Stanley Cavell à Graham Bradshaw, Lars Engle, Hugh Grady ou John Lee ${ }^{115}$, ont mis l'accent sur un «scepticisme», ou un «pragmatisme», qui exclurait «toute structure fixe de fait, de vérité et de connaissance» et ne reconnaîtrait que des valeurs «relativement stables» (mes italiques $)^{116}$. Or, si l'essayiste et le dramaturge ont des affinités incontestables dans leur reconnaissance de la diversité et du changement, ils en ont d'aussi profondes dans leur défense et illustration de valeurs constantes que je tiens pour « humanistes ».

Pour s'entendre sur une conception de l'humanisme au sens moderne, je prendrai pour point de départ la définition avancée par Tzvetan Todorov, dont Le jardin imparfait offre un panorama de la pensée humaniste depuis Montaigne et Descartes jusqu'aux temps présents. Écartant toute réduction à ce que l'on désignerait en anglais par simple «humaneness», il a proposé trois caractéristiques: l'autonomie du je, la finalité du tu, et l'universalité des ils ${ }^{117}$.

L'autonomie du «je » suppose un accord sur l'existence et la permanence $d u$ «je», question qui appelle un débat. La «finalité du tu» est une notion qui ne convient guère à l'auteur des Essais, principalement soucieux de s'observer, et chez un dramaturge on la repère malaisément. J'y substitue la «compréhension de l'autre», compréhension qui, chez Montaigne et chez Shakespeare, tend à l'identification plutôt qu'à la reconnaissance de l'altérité et de la «responsabilité pour l'autre» comme chez Emmanuel Lévinas prêchant l'Humanisme de l'autre hom me $e^{118}$. Or la « compréhension de l'autre» conduit à la compassion tout aussi bien que la «finalité du

\footnotetext{
114 Shakespeare and the Constant Romans, Oxford, O. U. P., 1996.

115 S. Cavell, Disowning Knowledge in Six Plays of Shakespeare, Cambridge, C. U. P., 1987; G. Bradshaw, Shakespeare's Scepticism, Brighton, Harvester, 1987; L. Engle, Shakespearean Pragmatism, U. of Chicago Press, 1993; H. Grady, Shakespeare's Universal Wolf, Oxford, O. U.P., 1996; J. Lee, Shakespeare's Hamlet and the Controversies of Self, Oxford, O. U. P., 2000

116 Voir Shakespearean Pragmatism, p. 86, 88, 102.

${ }^{117}$ Le jardin im parfait, Paris, Grasset, 1998, p. 48.

118 Paris, Fata Morgana, 1987, p. 86, 102-3, 109 ; cf. De Dieu qui vient à lídée, Paris, Vrin, 1998, p. 211-57.
} 
tu». On retrouve ainsi le sens moral, voire «humanitaire», de l'humanisme, sens écarté par Todorov, mais qui convient parfaitement à Montaigne lorsqu'il emploie le terme « inhumanité » $(\mathrm{I}, 14,53 \mathrm{c})$ pour dénoncer, avant le poète romantique Burns, «l'inhumanité de l'homme envers l'homme ». Shakespeare, je le montrerai, le rejoint sur ce point.

Reconnaître "l'universalité des ils », c'est reconnaître qu'on peut universaliser l'expérience individuelle, ce qui posera le problème de l'essence. C'est aussi admettre qu'il y ait des valeurs universelles et que des règles soient applicables à l'ensemble des hommes et des communautés à tout moment de l'histoire. À première vue, l'intérêt porté par Montaigne au pyrrhonisme, philosophie qui dénie à l'homme toute certitude, paraît exclure cette exigence. Mais cet in térêt s'exprime dans le cadre de cette "Apologie de Raymond Sebond» dont le but essentiel, Géralde Nakam l'a souligné, est de combattre tous les dogmatismes ${ }^{119}$. Montaigne s'y montre averti de la différence entre les sceptiques qui nient l'existence même de la vérité, et l'epoché des pyrrhoniens qui consiste à suspendre son jugement (II, 12, 544a). Mais il ne marque pas seulement sa préférence pour le pyrrhonisme; il va bien au delà, quand il admet qu'il existe une vérité dont «l'essence mesme... est uniforme et constante», vérité que «nous [la] corrompons et abastardissons par nostre faiblesse»(II, 12, 535a). Il déclare néanmoins que «nous sommes nais à quester la vérité », et considère le monde comme « une escole d'inquisition» (III, 8, 906b-c), car «il n'est desir plus naturel que le desir de connoissance... la verité est chose si grande, que nous ne devons desdaigner aucune entremise qui nous y conduise» (III, 13, 1041b). Certes, s'il propose aux lecteurs des « fantasies informes et irresolues », c'est, dit-il, « non pour establir la vérité, mais pour la chercher » (I, 56, 302a); cependant son but n'est pas l'ataraxie des Pyrrhoniens. Contrairement à Sextus Empiricus, observe William Hamlin ${ }^{120}$, il ne nous laisse pas sous l'impression qu'aucune connaissance certaine ne peut être atteinte — sauf, il faut l'admettre, dans certaines pages de l'Apologie. Encore faut-il noter que la phrase célèbre «Quelle vérité que ces montagnes bornent...?» (II,

\footnotetext{
119 Le dernier Montaigne, passim. Même point de vue dans une communication de W. Hamlin (Valence, 2001) qui constitue un chapitre d'un ouvrage à paraître: Engaging Doubt : Renaissance Scepticism and English Tragedy.

120 Voir note 11 .
} 
12, 563a) porte sur la diversité des lois et coutumes, non sur la distinction entre le vrai et le faux.

N'aurait-on pas exagéré l'influence du pyrrhonisme sur la pensée de Montaigne? Pyrrhon et les sceptiques ne sont mentionnés qu'une vingtaine de fois dans les Essais, et presque exclusivement dans l'Apologie (II, 12) où le scepticisme sert un dessein particulier ${ }^{121}$. Platon et le platonisme sont mentionnés 205 fois. Parfois, certes, d'un point de vue critique, en particulier à propos de la doctrine des Idées, mais le Platon de la République et des Lois est salué comme «maistre ouvrier en tout gouvernement politique » (III, 9, 930c). Lars Engle a reconnu que Shakespeare «traite le scepticisme même de façon plutôt sceptique ${ }^{122}$. De même, Montaigne semble y voir une forme de dogmatisme quand il montre que sa «plaisante science de l'ignorance » a inspiré à Pyrrhon des comportements absurdes (II, 29, 683a) ${ }^{123}$. Laisser son jugement en suspens n'implique pas nécessairement un ralliement au scepticisme intégral: Bacon y insistera dans la Préface du Novum Organum ${ }^{124}$.

Sans doute l'insistance de Montaigne sur la diversité des coutumes tout au long des Essais peut-elle conduire à un relativisme culturel et moral. Todorov l'admet, mais j'en suis moins convaincu. Constater une diversité, ce n'est pas nécessairement l'approuver. Que Montaigne, dans les circonstances tragiques des guerres de religion, en soit venu à considérer le conservatisme préférable à l'innovation ${ }^{125}$ ne signifie pas qu'il écarte la possibilité de concevoir des institutions et des lois plus justes. Qu'il déplore que la justice soit mal rendue implique même qu'une vraie justice pourrait exister (II, 12, 562a). Dans la traduction des Essais par Florio, Samuel Daniel avait loué la dénonciation de «ce tyran de l'univers, la coutume», qui conduit parfois, disait l'essayiste, à «forcer ... les reigles de nature» (I, 23,

${ }^{121}$ Pyrrhon ne réapparaît que deux fois : en II,20,655c, à propos de la vertu, et en II, 29, $683 \mathrm{a}$.

122 «Measure for Measure and Modernity: The problem of the sceptic's authority », dans Shakespeare and Modernity, éd. Hugh Grady, Londres et New York, Routledge, 2000, p. 86.

${ }^{123}$ En Angleterre Thomas Harriot raillait les «Sceptiques» qui, disait-il, «make there knowledge certayne to doubt assuredly : Ms cité par William Hamlin dans « Scepticism in Shakespeare's England », Shakespeare International Yearbook 2, Ashgate, 2002, p. 298.

$124 \mathrm{Il}$ y prend position entre deux extrêmes : «between the presumption of pronouncing on every thing, and the despair of comprehending anything».

125 Voir mon article «Self-consistency in Montaigne and Shakespeare » (note 1). 
106a). Mais il y a, dit Montaigne, des lois «désirables»: ce sont «les plus rares, plus simples et generales ${ }^{126}$, et «Nature les donne tousjours plus heureuses que ne sont celles que nous nous donnons. Tesmoing la peinture de l'aage doré des poëtes, et l'estat où nous voyons vivre les nations qui n'en ont point d'autres» (III, 13, 1042$43 \mathrm{~b})$. Il faudra revenir sur cette affirmation et la nuancer, mais c'est admettre implicitement l'existence d'un modèle idéal dont nos lois cependant s'éloignent «par leur desreiglement et deformité » en «leur dispensation et exécution» (III, 13, 1049b). Montaigne pourrait-il excuser « la desobeyssance» civile (III, 13, 1029-30b) et, en cas de choix douteux entre des lois contradictoires, conseiller l'abstention (III, 9, 972b), s'il n'avait la conviction que l'on peut choisir des voies meilleures?

Sur ce point je n'ai pas de parallèle précis à offrir chez Shakespeare. Mais la clémence, recommandée aux juges et aux monarques à la fois par l'humanisme antique et par l'humanisme chrétien, est une entorse à la loi ordinaire. Or, il n'est plus à démontrer que Montaigne et Shakespeare l'ont célébrée pareillement ; le second a même emprunté des arguments au premier dans Mesure pour Mesure et dans la Tempête : David Quint, après Eleanor Prosser, l'a souligné127.

Un obstacle à mon interprétation doit d'abord être écarté. La relativité des valeurs serait proclamée expressément par Montaigne dans deux essais et par Shakespeare dans Hamlet et dans Troilus and Cressida. Mais quand l'essayiste affirme «Que le goust des biens et des maux depend en bonne partie de l'opinion que nous en avons » (I, 14), il s'agit seulement de notre appréhension à l'égard de la mort, de la souffrance et de la misère ; le point de départ est d'ailleurs une citation d'Épictète. Et quand il écrit dans l'essai «Nous ne goustons rien de pur» (II, 20) que « la meilleure bonté ... a de la teinture vicieuse », c'est une observation à laquelle l'examen de conscience, et non le scepticisme, a souvent conduit les moralistes, chrétiens ou non.

\footnotetext{
126 Cette affirmation semble contredite par la constatation dans l'Apologie qu'il n'est pas «une seule» loi que l'on prétend «universellement reçeue» qui ne soit désavouée par plusieurs nations » (II,12,564a). À quoi l'on peut répondre : $1^{\circ}$ qu'il est ici question de lois existantes, non des lois qui pourraient être reconnues désirables ; $2^{\circ}$ que l'anti-dogmatisme de l'Apologie se voulait péremptoire et que la pensée de Montaigne est plus nuancée dans les autres essais.

127 Quint, Montaigne and the Quality of Mercy, Princeton U. P., 1998, chap. 2 ; Prosser, Shakespeare Studies 1 (1961), p. 221-26.
} 
Quand Hamlet dit «there is nothing either good or bad, but thinking makes it so» (II.ii.248-9), il utilise un proverbe ${ }^{128}$ pour expliquer que le Danemark est devenu pour lui une prison, non pour manifester son adhésion au scepticisme philosophique. La question de Troïlus «What's ought but as 'tis valued? » est suscitée par le mépris d'Hector à l'égard d'Hélène: «she is not worth what she doth cost the keeping ». Or, à nouveau l'idée est proverbiale ${ }^{129}$, mais ici un vrai débat s'engage. La réplique d'Hector n'écarte pas toute subjectivité du jugement, mais confère à la valeur une réalité objective : «value dwells not in particular will, / It holds his estimate and dignity / As well wherein 'tis precious of itself / As in the prizer» (II.ii.52-56). On peut penser que l'appel antérieur d'Ulysse au respect de l'ordre était dicté par les circonstances ${ }^{130}$, mais on ne peut douter de la sincérité d'Hector. Le plaidoyer de Trö̈lus pour l'honneur n'est pas moins sincère, car l'honneur avait une valeur en soi pour Shakespeare et ses contemporains. Grady présente ce dialogue comme « a diagnosis of the process of value creation in the commodification which is so relentlessly enacted ${ }^{131}$. Pourtant Hector voit clairement que les raisons avancées «fort superficiellement» (164) par Troïlus et Paris en faveur d'une «mauvaise cause» (116) ne sont pas un jugement objectif entre juste et injuste (169-70); la « loi de la nature» et celle des «nations » commandent de rendre Hélène à son époux. Son ralliement inattendu au point de vue de Troilus va, il le reconnaît, contre la "vérité » (truth) et paraît être dicté par le seul souci de préserver «our joint and several dignities» (192): les apparences extérieures de l'honneur. L'absurdité de cette guerre provoquée par un enlèvement et un adultère est évidente. Hector oublie la distinction faite par Montaigne : «Toute personne d'honneur choisit de perdre plustost son

\footnotetext{
128 Tilley, M 254, cité par Hibbard dans l'édition d'Oxford, Ham let (1987), p. 216.

129 Tilley et Dent W923, cités par Spriet et Monsarrat dans William Shakespeare, Tragicomédies, vol. I (Paris, Robert Laffont, 2002), p. 129, édition utilisée pour les références.

130 Jonathan Dollimore soutient que cet appel au respect d'un ordre qui serait inscrit dans la loi naturelle est contredit par la constatation du désordre qui règne dans la société (Radical Tragedy, Harvester-Wheatsheaf, 2e éd., 1984, p. 43), mais ce contraste entre la loi de nature et le comportement humain était universellement admis et précisément invoqué pour rappeler la créature à ses devoirs.

${ }^{131}$ Shakespeare's Universal Wolf, p. 93-4. - Terry Eagleton prétend que Hector ne peut opposer au subjectivisme de Troïlus que «simply a wider intersubjectivity» (William Shakespeare, Oxford, Blackwell, 1986, p. 60), argument écarté par Richard Strier dans Resistant Structures, University of California Press, 1995, p. 63-4.
} 
honneur, que de perdre sa conscience» (II,16,614c). Troïlus est dans l'erreur quand il exalte la valeur d'Hélène. C'est clairement suggéré par le recours à la même métaphore de la «perle» inestimable à propos d'Hélène et de Cresside (I.i.93 ; II.ii.81) : l'une et l'autre sont des perles fausses et Troïlus s'abuse. Othello s'abusera à l'inverse en jetant la perle vraie (v.ii.347). Dans Troilus and Cressida Shakespeare a bien «démystifié les codes de valeur» comme l'écrit Engle ${ }^{132}$, mais il ne les a pas subvertis.

Montaigne, de son côté, tout au long des essais, invoque des valeurs morales telles que la loyauté et le respect de la parole donnée (II, 17, 630a ; III, 9, 944b). Il exige la véracité dans la vie publique et privée (II, 18, 649a). S'il reconnaît que « la vérité et le mensonge ont leurs visages conformes », ce n'est pas pour les confondre, mais pour déplorer notre tendance à «nous embrouiller» (III, 11, 1004b). Il estime la bonté « loing au delà » de la valeur guerrière chez le premier Scipion dont il loue la «debonnaireté et humanité» (III, 9, 948c). Même en son exposé théorique de nos ignorances dans l'Apologie, ce qui est dit hors d'atteinte, ce sont les certitudes de la connaissance, intellectuelle et sensible, mais non les convictions morales: les «valeurs humanistes» reconnues dans l'éthique antique comme dans l'éthique chrétienne ne sont pas mises en question. Montaigne a sa perspective et ses préférences personnelles, mais, sans nier l'influence $\mathrm{du}$ réalisme de Machiavel, on peut soutenir que, contrairement à l'auteur du Prince, l'auteur des Essais ne rejette pas ce que Victoria Kahn appelle «the Ciceronian and humanist equation between honestas and utilitas » ${ }^{133}$. Et il n'a pas de sympathie profonde pour la virtus, quand celle-ci se manifeste seulement sous ses formes guerrières ou stoïques, inhumaines envers soi-même comme envers les

\footnotetext{
132 Shakespearean Pragmatism, p. 49. La thèse générale de Engle est bien résumée par Grady : le pragmatisme de l'écrivain se pliant aux variations d'un marché intellectuel: « the disparateness and multiple perspectives of Shakespearean drama and poetry can best be understood as instances of a methodological 'pragmatism' in which the competing values and ideologies of this transitional period are put into circulation in a market-like free play of intellectual contestation » (Shakespeare's Universal Wolf, p. 36).

133 Victoria Kahn, «Virtù and the Example of Agathocles in Machiavelli's Prince » dans A. R. Ascoli and Victoria Kahn, Machiavelli and the Discourse of Literature, Cornell U. P. 1993, p. 198 ; voir Hugh Grady "Shakespeare's Links to Machiavelli and Montaigne: Constructing Intellectual Modernity in Early Modern Europe », Comparative Literature 52 (2000), p. 122
} 
autres; sur ce point les arguments de D. L. Schaeffer sont irréfutables ${ }^{134}$.

Dans l'examen de ces valeurs humanistes, privilégiées à la fois par Montaigne et par Shakespeare, partons de la « compréhension de l'autre». Celle-ci peut se manifester dans une relation personnelle d'amitié, thème favori des humanistes de la Renaissance. Montaigne, à propos de La Boétie, assure l'avoir poussée jusqu'à une "parfaite et entiere communication » (II, 8, 376a). Cette amitié, dit-il, « ayant saisi toute ma volonté, l'amena se plonger et se perdre dans la sienne; qui, ayant saisi toute sa volonté, l'amena se plonger et se perdre en la mienne ${ }^{135}$. Cette même volonté de se perdre en l'autre anime le poète des Sonnets, bien qu'il ne la retrouve que momentanément, ou illusoirement, chez le jeune homme aimé. Montaigne et Shakespeare élèvent chacun un mémorial à l'ami dont l'un est séparé par la mort, l'autre par la vie ${ }^{136}$. Mais chacun d'eux élève du même coup un «mémorial» à soi-même : "My life hath in this line some interest, / Which for memorial still with thee shall stay » (Sonnet 74).

$\mathrm{Au}$ delà des relations d'amitié, ils étendent l'un et l'autre leur sympathie à des hommes de basse condition. Certes, ils étaient de leur temps et les titres de noblesse gardaient de la valeur à leurs yeux. Montaigne ne se contente pas d'être magistrat, mais prétend appartenir à la «noblesse d'épée »; Shakespeare use de sa célébrité naissante pour obtenir que son père soit élevé au rang de gentilhomme. Mais si le dramaturge manifestement abhorre la versatilité et la cruauté des foules, en revanche, quand il met en scène des individus, il peint avec sympathie les gens du commun, avec compassion les miséreux, comme le mendiant nu dans King Lear. Quant au seigneur de Montaigne, il déclare avoir «veu en [son] temps cent artisans, cent laboureurs, plus sages et plus heureux que des recteurs de l'université, et lesquels [il] aimer[ait] mieux ressembler» (II, 12, 466b). C'est, dit-il, parmi les gens de basse condition qu'il «n'est pas si nouveau d'y voir quelque traict de rare bonté»; «les exemples de vertu se logent rarement » chez les nobles et chez les riches (II, 25, 724a).

\footnotetext{
134 Voir The Political Philosophy of Montaigne (Cornell U. P., 1990), p. 232 et suiv. 135 I, 28, 187c. Cf. III, 9, 954-55b.

${ }^{136}$ Dans son Essai sur les Essais (Paris, Gallimard, 1968) Michel Butor les présente comme un «monument » pour La Boétie.
} 
La compréhension de l'autre s'étend à l'étranger quand Montaigne proclame : "j'estime tous les hommes mes compatriotes, et embrasse un Polonois comme un François» (III, 9, 950b). Shakespeare, dans un dessein comique, se moque des travers des Espagnols, des Français, des Gallois; mais sans animosité, à la différence des satiriques et des pamphlétaires de son temps. Qui plus est, il a sans doute écrit la scène où Sir Thomas More adjure les émeutiers d'épargner les étrangers établis à Londres, les invitant à s'imaginer eux-mêmes en butte à semblable «inhumanité » s'ils vivaient dans un autre pays que le leur ${ }^{137}$.

Cette compréhension englobe les étrangers les plus éloignés par la race ou la religion. Dans Othello, Shakespeare donne à un Maure le rôle le plus noble malgré son manque de jugement. Sans renoncer à certains stéréotypes, il prête à l'usurier juif Shylock, le représentant de la communauté la plus méprisée de son temps, un discours sur l'universalité de la condition humaine qui peut s'appliquer à toutes les situations, toutes les inimitiés entre ethnies, nations ou communautés. On connaît ce discours qui aboutit à cette amère constatation : " Si un Juif fait injure à un Chrétien, celui-ci le supporte-t-il humblement ? Vengeance! Si un Chrétien fait injure à un Juif, comment celui-ci devrait-il le supporter à l'exemple des Chrétiens? Eh bien! vengeance !» (III.i.44-53). Si Shylock est néanmoins condamné à la fin de la pièce, c'est qu'il a rejeté l'appel de Portia à la miséricorde (IV.i.178 et suiv.). À l'inverse, dans The Tempest, Prospéro, chassé de son duché, menacé de mort dans son île, refuse la vengeance : «The rarer action is / In virtue than in vengeance» (v.i.27-8). Ce pardon, il l'étend à tous ceux qui l'ont exilé ou qui ont voulu le tuer, y compris Caliban.

Or Caliban - la figure de «l'autre» sous l'aspect du sauvage, comme l'a dit Jean-Marie Maguin ${ }^{138}$ — est décrit d'abord comme «a freckled whelp...not honour'd with a human shape », mais on découvre qu'il n'est pas en dehors de l'humanité pour Shakespeare, comme le furent longtemps les Indiens d'Amérique aux yeux de certains conquistadors et de certains théologiens (je songe à la Controverse de Valladolid). Prospéro ne finit-il point par le reconnaître quand il dit «This thing of darkness I / Acknowledge mine» (v.i.275-6) ? Caliban est vu comme un «monstre» par des ivrognes (II.ii.65), mais

${ }^{137}$ Histoires, vol. II, p. 800-02.

138 Dans «The Tempest and Cultural Exchange », Shakespeare Survey 48 (1995), p. 149. 
Montaigne, dans l'essai «D'un enfant monstrueux», rappelle que les monstres sont aussi l'œuvre de Dieu et que «nous appellons contre nature ce qui advient contre la coutume » (II, 20, 691c). Caliban n'obéit qu'à l'instinct, mais, asservi, il revendique le droit à la liberté et à la possession d'une île qui lui appartenait avant l'arrivée du colonisateur. Cependant Shakespeare ne l'idéalise pas, comme Montaigne apparemment idéalise les cannibales (I, 31), et le rêve utopique de Gonzalo, dont le royaume «surpasserait l'âge d'or », ne résiste pas à l'ironie des réalistes (II.i.132-57).

L'âge d'or ne peut renaître. Montaigne, lui, paraît admettre qu'il avait survécu en cette nation d'Amérique «en laquelle il n'y a aucune espèce de trafique ; nulle cognoissance de lettres; nulle science de nombres; nul nom de magistrat, ny de superiorite politique ; nul usage de service, de richesse ou de pauvreté; nuls contrats; nulles successions; nuls partages; nulles occupations qu'oisives; nul respect de parenté que commun; nuls vestemens; nulle agriculture; nul metal; nul usage de vin ou de bled. Les paroles mesmes qui signifient la mensonge, la trahison, la dissimulation, l'avarice, l'envie, la detraction, le pardon, inouïes. Combien [Platon] trouveroit-il la republique qu'il a imaginée esloignée de cette perfection!» (I, xxxi, 204a). Mais, la mention de Platon le rappelle, l'Utopie était alors un genre favori et un rêve d'humaniste. Montaigne n'affirme rien puisqu'il écrit «il me semble [mes italiques] que ce que nous voyons par experience en ces nations là, surpasse non seulement toutes les peintures dequoy la poësie a embelli l'age doré, mais encore la conception et le desir mesme de la philosophie» (ibid.) Peinture embellie, conception née du désir? Mais le véritable humanisme, même lorsqu'il n'est pas chrétien, sait que tout homme est soumis à des pulsions violentes et dangereuses, que celles-ci procèdent d'une Chute mythique ou de l'id freudien. Ce que Montaigne reconnaît, tout comme Shakespeare, c'est que les civilisés ne sont pas moins barbares que les barbares, mais «chacun appelle barbarie ce qui n'est pas de son usage » (I, 31, 203a). Cependant, si Montaigne « se sert de l'image des cannibales pour critiquer notre propre société » ${ }^{139}$, comme Todorov l'observe, il «ne se départit pas de sa préférence pour les civilisations

${ }^{139}$ Cf. William Hamlin, The Image of America in Montaigne, Spenser, and Shakespeare : Renaissance Ethnography and Literary Reflection, New York, St Martin's Press; Basingstoke, Macmillan, 1995, p. 63 
grecque et romaine [...]; il n'estime donc les cannibales que dans la mesure où ceux-ci ressemblent aux Grecs et aux Romains : bravoure, déférence envers les femmes, poésie anacréontique» ${ }^{140}$. Frank Lestringant note aussi que les valeurs accordées aux Indiens les «situent dans l'héritage direct des grands hommes de l'Antiquité » ${ }^{141}$ et Marc Fumaroli insiste sur ce «constant rapprochement avec l'antiquité gréco-romaine» dans l'essai «Des coches» comme dans l'essai « Des cannibales » ${ }^{142}$.

Certaines interprétations et mises en scène du théâtre de Shakespeare ne présentent aujourd'hui qu'une image barbare et une vision pessimiste de l'humanité. Sous l'influence d'Antonin Artaud et du livre de Jan Kott, Shakespeare notre Contemporain, on transforme la tragédie shakespearienne en un théâtre de la cruauté alors que la pitié y est toujours présente, comme dans la tragédie grecque et la poétique d'Aristote ${ }^{143}$. King Lear est exemplaire à cet égard. Dans une représentation qui fit école, Peter Brook a supprimé la scène où Cordélia est évoquée comme l'emblème de la pitié (IV.iii), ainsi que le passage où, après le supplice de Gloucester, des serviteurs expriment leur indignation et vont chercher de quoi «panser son visage en sang » (III.vii.fin). Ces textes, il est vrai, ne figurent pas dans l'in-folio de 1623 et Shakespeare lui-même a pu être l'auteur d'une révision qui les fit disparaître. Mais j'ai montré dans un commentaire sur cette tragédie ${ }^{144}$ que, même après ces coupures, il subsiste dans la pièce une quinzaine d'appels directs à la compassion. D'autres metteurs en scène ont voulu

\footnotetext{
140 Nous et les autres, Paris, Seuil, 1989, p. 298, 302-3. Selon Claude Lévi-Strauss dans Histoire de Lynx (Paris, Plon, 1991, p. 281) Montaigne navigue entre deux écueils : l'utopie, qui donne à une société une assise rationnelle, et le relativisme culturel, qui impose "le rejet de tout critère absolu dont une culture pourrait s'autoriser pour en juger une autre». À mon sens il rejette l'utopie, mais ne se résigne pas au relativisme.

${ }^{141}$ Le Cannibale, Paris, Perrin, 1994, p. 188. Il est possible que Shakespeare n'ait pas eu conscience de la complexité de la position de Montaigne et qu'il raille son "radicalism" comme le pense Jean-Marie Maguin (Shakespeare Survey 48, p. 153), mais on ne peut aller jusqu'à affirmer comme Jonathan Bate que "Shakespeare has ... reversed Montaigne » (Shakespeare and Ovid, Oxford, O. U. P., 1993, p. 256).

142 «Les Anciens du Nouveau Monde », Montaigne et le Nouveau Monde, éd. C. Blum, M. L. Demonet, A. Tournon, Société Internationale des Amis de Montaigne, SPEC, 1994, p. 26 7 .

143 Shakespeare n’a probablement connu que la tragédie sénéquéenne, mais les grands auteurs se rejoignent au sommet.

144 Voir ma Notice sur Le Roi Lear dans Shakespeare, Euvres complètes, Tragédies, vol. II, Gallimard, coll. «Pléiade », 2002, p. 1381.
} 
effacer toute distinction entre le fils dénaturé, Edmond, et le fils aimant, Edgar, prêtant à ce dernier dans le duel qui oppose les deux frères une sauvagerie qui n'est pas dans le texte. À propos de la fin de la tragédie on a parlé de «nihilisme»; pourtant, autour de Lear, coupable racheté, et de Cordélia, victime innocente, on voit se rassembler symboliquement les survivants, Edgar, Kent et Albany, qui ont illustré les valeurs de loyauté, de respect des liens naturels et sociaux, de courage et de compassion ${ }^{145}$. Dans une autre tragédie l'imagination hallucinée de Macbeth lui fait entrevoir avant le meurtre de Duncan «pity; like a naked new-born babe / Striding the blast» (I.vii.21-25). Quoique devenu un tyran sanguinaire, Macbeth n'est pas aveuglé sur lui-même au point de ne plus pouvoir discerner les vraies valeurs: "that which should accompany old age, / As honour, love, obedience, troops of friends, / I must not look to have...» (v.iii.26-28). L'auteur des pièces historiques sur les Guerres des deux Roses n'avait vu que démence dans les guerres civiles: «England hath long been mad, and scarred herself; / The brother blindly shed the brother's blood; / The father rashly slaughtered his own son » (Richard III, v.viii.23-25), comme dans 3 Henry VI, où un fils à son insu tue également son père (II.v.61-93). Que de lamentations sur les innocents assassinés, en particulier les enfants, dans ces drames historiques! Combien d'appels à la compassion lancés par les témoins de ces atrocités $^{146}$ ! Shakespeare n'hésite pas dans King John à faire du meurtre du jeune Arthur le motif essentiel de la révolte des barons, et cela sans fondement historique.

Or Montaigne, qui eut en France l'expérience directe d'atroces guerres civiles entre catholiques et protestants, a dénoncé de même «l'horrible corruption de meurs que les guerres civiles apportent » (I, 23, 119b). «Monstrueuse guerre», s'écrie-t-il ailleurs, «les autres agissent au dehors; cette-cy encore contre soy se ronge et se desfaict par son propre venin» (III, 12, 1018b). Il manifeste comme Shakespeare une aversion spontanée envers la cruauté, consacre un essai à la dénoncer (II, 11) et revient souvent sur ce thème, notamment à propos des supplices infligés aux Indiens d'Amérique ${ }^{147}$. Dans $L e$

\footnotetext{
145 Ibid., p. 1373

146 Voir notamment 3 Henry VI, I.iii ; I.iv.138-172 ; Richard III, IV.iv.9-135.

${ }^{147}$ Voir II, 27, 679-80a, III, 6, 889b et suiv.
} 
dernier Montaigne Géralde Nakam parle de «l'immense procès de la cruauté humaine dressé dans les Essais » ${ }^{148}$.

Cette aversion envers la cruauté, Shakespeare l'étend à la cruauté envers l'animal, qu'il s'agisse du lièvre pourchassé dans Venus and Adonis, ou du cerf expirant dans As You Like It. Or, l'essayiste s'était apitoyé aussi sur le cerf (II, 11, 412a) et il s'était indigné que ce soit «passetemps aux meres de veoir un enfant tordre le col à un poulet et s'esbattre à blesser un chien et un chat» (I, 23, 107b). Quand la mère de Coriolan se réjouit des dispositions martiales de son petitfils qui «massacre un papillon» (« how he mammocked it !», I.iii.67), Shakespeare à mon sens dénonce obliquement la part de fureur instinctive qui entre dans l'héroïsme de son père. Dès le Moyen Âge Jean de Salisbury s'était attaché à démontrer que le massacre des animaux par l'homme le ramenait lui-même à la bestialité et la critique de la chasse par Érasme et par Sir Thomas More était un topos spécifique de l'humanisme ${ }^{149}$.

Un problème n'est pas résolu. Sur quoi fonder ce refus de la cruauté ? Ni Montaigne, ni Shakespeare ne prétendent que l'homme est naturellement bon. L'essayiste observe que Nature a «elle mesme attaché à l'homme quelque instinct à l'inhumanité» (II, 11, 412b) ; comme Lucrèce, il admet qu'on peut trouver du plaisir à voir les autres souffrir (III, 1, 768b). Il ne sait lui-même si sa haine de la cruauté est due principalement à l'hérédité, ou à son éducation, ou à une disposition innée qu'il qualifie même de «mollesse». Pourtant il conclut qu'il la hait «et par nature et par jugement» comme «l'extreme de tous les vices»(II, 11, 408a). Par quel jugement? Il n'invoque pas à ce propos les enseignements du christianisme - et pour cause, en ces temps de guerres de religion! Il semble admettre que les lois de la conscience prennent souvent leur origine dans la coutume ${ }^{150}$, et apparemment ne se fie qu'à une «conscience bien reglée » (II, 16, 607a). Comment peut-elle l'être ? Ni par la nature seule, qui parfois pousse à l'inhumanité, ni par la religion seule, car il voit «une distinction enorme entre la devotion et la conscience ${ }^{151}$. Le

\footnotetext{
148 Le dernier Montaigne, p. 65 ; cf. p. 71-78, 153-54

${ }^{149}$ Voir Claus Uhlig, "The Sobbing Deer: As You Like It, II.i.21-66 and the Historical Context », Renaissance Drama, n.s. III (1970), p. 79-109.

150 Schaeffer y insiste, op. cit, p. 328-29.

151 III, 12, 1037c. - Dans le même passage il invoque la « raison universelle empreinte en tout homme non desnaturé », ce qui n'est guère conséquent : il fait tantôt appel à la nature
} 
sentiment seul devrait-il inspirer ce refus de la cruauté ? Mais comment le généraliser si ce sentiment n'existe que chez certains ? Sans doute en s'appuyant sur la «compréhension de l'autre », qui est possible pour tout homme s'il fait l'effort de s'imaginer à la place de celui qui souffre; effort d'imagination dont Montaigne s'est déclaré capable tout autant qu'un dramaturge ${ }^{152}$. Dans L'humanisme de l'autre homme, Emmanuel Lévinas s’interroge: "Est-il certain que déjà l'expérience la plus humble, de celui qui se met à la place de l'autre c'est-à-dire s'accuse du mal ou de la douleur de l'autre — n'est pas animée du sens le plus éminent selon lequel 'je est un autre'? 153

$\mathrm{J}$ 'en viens enfin à la caractéristique première de l'humanisme. Ces valeurs morales doivent être reconnues et assumées par un «je» qui exerce son jugement de façon constante avec une certaine liberté. Encore faut-il que ce «je » existe. Sur le plan théorique, j'en ai débattu dans un livre, Seven Metaphysical Poets - A Structural Study of the Unchanging Self ${ }^{154}$. Dans le cas particulier de Montaigne je peux renvoyer aux substantielles analyses de Jean Starobinski ${ }^{155}$ et à mes brèves remarques dans "Self-consistency in Montaigne and Shakespeare». J'ajoute au passage que l'autonomie du «je» justifie une insistance sur le libre arbitre conforme à la théologie de Sebond ${ }^{156}$. Montaigne s'est reconnu libre de gouverner ses impulsions, de se contrôler et de se former, pour faire enfin émerger sa «maistresse forme ».

Mais l'auteur de l'Apologie échappe-t-il à toute contradiction ? Ce n'est pas évident à première vue. Le jugement doit s'appuyer sur la raison. Or Montaigne dénonce les égarements de la raison humaine. Cependant, comme Lévi-Strauss l'a noté ${ }^{157}$, il y fait sans cesse appel. La raison humaine est faillible, mais elle fait partie de la nature de l'homme : «la semence de la raison universelle [est] empreinte en tout

contre la raison raisonnante, tantôt appel à la raison, pour corriger les instincts mauvais mis en nous par la nature. De même qu'on ne rencontre une conscience bien réglée que chez certains individus, de même la bonté est le privilège d'une «nature bien née » (III, 2, $784 \mathrm{~b})$.

152 I, 21, 95c ; 26, 176b ; III, 4, 816c ; cf. « Self-consistency in Montaigne and Shakespeare ».

153 Op. cit., p. 103.

154 Oxford, O. U. P., 2000 ; voir l'Introduction.

155 Montaigne en mouvement, chap. VI « Chascun est aucunement en son ouvrage».

156 Voir E. Colomer, «Raymond Sebond, un humaniste avant la lettre», Montaigne, Apologie de Raymond Sebond, De la Theologia à la Théologie, éd. C. Blum, Paris, Champion, 1990, p. 60-61.

157 «En relisant Montaigne» dans Histoire de Lynx (Plon, 1991), p. 280. 
homme non desnaturé» (III, 12,1037c). Le paradoxe est que la raison seule permet à l'homme de ne pas «se laisser tyranniquement emporter» par la nature quand la nature l'entraînerait au mal (II, 8, 366a). Donald Frame a proposé de distinguer entre la raison raisonnante (je préfèrerais «spéculative»), celle des philosophes et des théologiens, dont les errements sont ridiculisés, et une raison raisonnable, qui semble opérer essentiellement «dans le domaine de la conduite humaine ${ }^{158}$. Cette reasonableness me paraît s'apparenter $\mathrm{au}$ «common sense », au sens où l'entendront Reid et l'école écossaise $\mathrm{du} \mathrm{XVIII}^{\mathrm{e}}$ siècle, opposant la réalité de la perception immédiate et les vérités du sens commun à la fois à l'idéalisme de Berkeley et au scepticisme de Hume. Mais il faut à mon sens y adjoindre une troisième "raison ", celle qui procède à un examen objectif des faits, à la manière de la science expérimentale mais sans théoriser, ou bien encore à la manière du magistrat que fut Montaigne ${ }^{159}$. Typique est son attitude à l'égard de l'histoire. Il reconnaît que dans ce domaine la recherche de la vérité est «délicate», mais il n'assimile pas les récits historiques à des fictions comme Hayden White, ni ne considère les faits comme des « interprétations », ou des «signifiants », à la manière de Nietzsche, de Roland Barthes ou de Michel de Certeau ${ }^{160}$. La vérité peut être accessible si, dit-il, «à la mode d'une information judiciaire», on «confronte les tesmoins et reçoit les objects sur la preuve des pontilles de chaque accident » (II, 10, 398a). L'existence de la vérité n'est pas niée, mais, comme disait Donne, "hee that will / Reach her, about must, and about must goe» («Satyre III»). À cet égard Montaigne contredit expressément Sextus Empiricus pour qui tout jugement impartial est impossible ${ }^{161}$.

Tout en admettant qu'il peut nourrir lui-même «une opinion fauce » (III, 13, 1051b), Montaigne a donc une certaine confiance en son propre jugement ${ }^{162}$ et sa «capacité de trier le vray» (II, 17, 641a) quand on considère simplement des faits sans prétendre « en penetrer

158 «Montaigne's Dialogue with his Faculties », French Forum 1 (1976), p. 203.

159 Sebond mettait aussi l'accent sur la vérité établie «per experientiam» ou par "témoignage".

160 Voir mon article «Literary History and the Search for Certainty d dans New Literary History 27 (1996), p. 531 et suiv.

${ }^{161}$ Voir Esquisses pyrrhoniennes, éd. P. Pellegrin, Paris, Seuil, 1997, I, 14, [135-140], p. 129-31.

${ }^{162}$ Sur le rôle du jugement voir R. C. La Charité, The concept of judgment in Montaigne, La Haye, 1968. 
l'origine et l'essence » (III, 11, 1003c). Nous avons l'usage des choses, mais la connaissance des causes appartient à Dieu (III, 11, 1003). Dans le domaine restreint de l'expérience ce «sceptique» martèle des convictions; sous sa plume reviennent sans cesse les expressions « en vérité », « de vrai», « il est vrai », « il est bien vrai», bien est vrai que... » 163

Or un lien naturel s'établit entre le «vrai» et l'« essence» : ainsi « la créance se donne essence et vérité » (I, 14, 67c), «l'essence et verité de la chose » apparaissent conjointement (II, 6, 352a) ${ }^{164}$. Essence et réalité sont aussi associées : on «possede ses biens par fantasie, les maux en essence » (II, 12, 468b) ; une action vertueuse en apparence peut n'en avoir pas «l'essence» $(\mathrm{I}, 37,226 \mathrm{a})$; le fard peut « corrompre l'essence des choses » $(\mathrm{I}, 51,292 \mathrm{a})^{165}$. La pen sée de Montaigne n'est pas « anti-essentialiste». Sa position, me semble-t-il, se clarifie, si l'on retient une distinction qui sera clairement formulée par Locke: «essence» est pris par Montaigne en son sens premier, essentia, qui désigne l'essence réelle d'une chose particulière, «the very being of anything ${ }^{166}$, sa nature et sa constitution interne. Toute «chose»a donc une essence ${ }^{167}$ pour l'auteur des Essais, qui sur ce point suit Aristote dont il est souvent proche comme l'a démontré Michael Screech ${ }^{168}$. Mais il ne s'agit pas de l'essence «logique» qui constitue un genre et que Locke dit «nominale », lui refusant toute existence en dehors du langage : Montaigne pense comme les juristes ${ }^{169}$ et comme les nominalistes ${ }^{170}$ qu'il faut prêter plus d'attention aux choses qu'aux

163 Voir « vérité » et « vrai » dans la Concordance de Leake. Il s'agit parfois de confirmer un doute, mais les assertions abondent. Cependant cette insistance n'est pas dogmatique et se concilie avec le recours non moins fréquent à des expressions qui «amolissent et moderent la temerité de nos propositions : A l'avanture, Aucunement, Quelque, Ont dict, Je pense, et semblables» (III, 11, 1007b).

164 Cf. « veritable essence », II, 12, 579a.

165 Sur l'opposition entre l'essence et l'apparence, ou «semblance », voir aussi II, 12, 544a, III, $5,866 \mathrm{c}$.

${ }^{166}$ Essay concerning Human Understanding, III, iii, 15 et suiv. Montaigne emploie aussi l'expression «essence réelle» (III, 10, 1011b)

167 Voir «l'essence des choses » (I, 51, 292a) ; « les choses... selon la loy de leur essence » (II, 12, 572a), etc.

168 Métaphysique, IV, c, 4 ; Screech, Montaigne and Melancholy, Londres, Duckworth, 1983, voir l'Index.

169 Ian Maclean, Montaigne philosophe (Paris, PUF, 1996), p. 46.

170 «Il y a le nom et la chose...»: II, 16, 601a. Sur Montaigne et le nominalisme voir Antoine Compagnon, Nous, Michel de Montaigne, Paris, Seuil, 1980 
mots. Il s'agit encore moins de l'essence idéale, élevée au rang d’une réalité transcendante.

La tendance actuelle est de rejeter toute présence d'un «essentialisme»dans la pensée de Montaigne et de Shakespeare ${ }^{171}$. Ce n'est admissible que si l'on songe uniquement à un essentialisme de type platonicien. Contrairement à Pyrrhon, pour qui les choses n'ont pas d'essence ${ }^{172}$, Montaigne admet la réalité des essences particulières ; mais il considère, comme Locke, que notre examen des choses ne peut « en penetrer l'origine et l'essence » (III, 11, 1003c), car toute essence « est abstruse et occulte» (III, 2, 791b). Cela dit, il n'est pas certain que celui qui se présente lui-même comme un «philosophe impremedité et fortuite $($ II, 12, 528c) fasse toujours un emploi rigoureux des termes, qu'il s'agisse de l'essence ou de la forme. Ce qui me paraît significatif, c'est qu'il admette la difficulté de connaître les essences et néanmoins, à propos de son auto-portrait, en vienne à proclamer : « Ce ne sont mes gestes que j'escris, c'est moy, c'est mon essence» (II, 6, 359c). Non seulement le moi aurait bien une essence, mais on pourrait la découvrir et la coucher sur le papier ! À quoi certains répondent : ce «moi » n'est que traces sur le papier. Ce qui est, de toute évidence, vrai pour le lecteur, en dehors même de toute théorie, mais il n'y aurait pas eu ces traces si ce moi n'avait pas existé.

Dans la célèbre phrase «chaque homme porte la forme entière de l'humaine condition »(III, 2, 782b), on remarquera que Montaigne n'a pas écrit «la forme de l'homme», mais la «forme» de sa «condition». Il ne veut pas seulement dire qu'on retrouve en tout homme la «forme» universelle qui distingue l'espèce humaine de l'animal ou de l'ange. Dans le contexte il affirme qu'une vie «privée », propre à un individu, peut porter témoignage de toutes les possibilités offertes à chaque homme par «l'humaine condition ${ }^{173}$. Le particulier et l'universel sont étroitement associés, mais l'essence, la réalité de

\footnotetext{
${ }^{171}$ Grady reconnaît toutefois qu'en certains passages leur pensée «appears much less assimilable to Postmodernist claims for a consistent early modern anti-essentialism» : « Shakespeare's Links to Macchiavelli and Montaigne», p. 131-32.

172 Ian Maclean, op. cit., p. 50.

${ }^{173}$ Hamlin dans The Image of America suggère que Montaigne se prétend capable d'éprouver imaginativement tout ce que les hommes peuvent éprouver, «totalizing in his own individual form all the forms of mankind» (p. 63); c'est peut-être aller trop loin. Sur l'utilisation du mot «forme»par Montaigne, voir «Self-consistency in Montaigne and Shakespeare».
} 
l'existence, est presque toujours située dans l'individuel ${ }^{174}$ : d'où l'accent mis sur la différence et la diversité, comme l'a souligné André Tournon ${ }^{175}$. La nature des hommes, observe Todorov, c'est précisément «leur capacité d'avoir une culture, une histoire, une identité individuelle; leur nature consiste à n'être pas entièrement déterminés » ${ }^{176}$. À la limite, on peut donc comme Maclean assimiler cette absence de détermination à une «absence d'essence et de substance» qui «devient par là son propre contraire, à savoir, la substance et l'essence humaines ${ }^{177}$. Je pense néanmoins que Montaigne se peint avec beaucoup trop de précision pour qu'on réduise son essence à une absence de détermination. Malgré l'influence des sceptiques il se rattache à une autre lignée dans l'observation de soi. Dans sa traduction de Sebond il avait écrit: "il n'y a rien de plus familier, plus interieur, \& plus propre à chacun que soy-mesme à soy » ${ }^{178}$. C'est déjà du pur Montaigne, mais c'est aussi le prolongement de la tradition augustinienne ${ }^{179}$.

Shakespeare emploie métaphoriquement le mot essence quand Valentin dit de Sylvia qu'elle est son essence (Two Gentlemen of Verona, III.i.182). Dans Measure for Measure (II.ii.123) où glassy essence souligne la fragilité de l'être humain, « essence » peut être pris au sens premier d'essentia. Dans Othello, quand Iago dit «Her honour is an essence that's not seen »(IV.i.16), l'ironie consiste à suggérer que cette essence d'un honneur particulier, celui de Desdémone, essence normalement invisible, est en réalité absente. Même l'essence d'un amour défini en termes métaphysiques dans le poème "The Phoenix and Turtle » reste l'essence réelle d'un être singulier, à la fois double et indivis. Et si toutes les grâces s'incarnent en l'aimé dans le sonnet 53, ce n'est pas à la façon dont la Beauté idéale des platoniciens s'incarnait en la femme adorée pour Pétrarque ou Sidney: toutes ces grâces sont contenues dans la «substance» de l'être aimé.

174 Il lui arrive, certes, d'évoquer «nostre essence... imparfaicte» (II, 16, 601a), ce qui implique une essence commune à tous les hommes, mais c'est pour l'opposer à l'essence divine.

175 Voir Montaigne et le Nouveau Monde, p. 252

176 Le jardin imparfait, p. 82.

177 Montaigne philosophe, p. 83.

${ }^{178}$ La Théologie Naturelle de Raymond Sebond, Traduicte par messire Michel, Seigneur de Montaigne (Paris, 1581), chap. 1, f $5 \mathrm{r}^{\circ}$

${ }^{179}$ Voir Genèse de la conscience moderne, éd. R. Ellrodt, Paris, PUF, 1983, « De Platon à saint Augustin », p. 40-48. 
Que Montaigne et Shakespeare ne conçoivent pas l'essence à la manière de l'idéalisme platonicien ne signifie point qu'ils n'aient pu avoir une certaine expérience de la transcendance ; la transcendance se conçoit aussi de différentes façons. L'aspiration stoïcienne et humaniste à s'élever au dessus de soi-même, formulée par Sénèque ${ }^{180}$ et reprise par le poète élisabéthain Samuel Daniel, peut être considérée comme un effort de l'homme pour se dépasser: "unlesse above himselfe he can / Erect himselfe, how poore a thing is man!»181. Or Montaigne la récuse expressément: "Voylà un bon mot et un utile desir, mais pareillement absurde» (II, 12, 588a). Contrairement à Sebond il ne pense pas qu'on puisse « enjamber de l'homme jusques à Dieu ${ }^{182}$ : sa conscience «se contente de soy, non comme de la conscience d'un ange ou d'un cheval, mais comme de la conscience d'un homme» (III, 2, 784c). Il déclare « je puis desirer en general estre autre», mais ne fait point effort pour changer: «Je ne puis faire mieux» (III, 2, 791b). Shakespeare dans les Sonnets consent aussi à être ce qu'il est : «I am that I am » (Sonnet 121) ${ }^{183}$.

Cependant, Montaigne, à travers sa «parfaite amitié » pour La Boétie, Shakespeare, dans sa conception d'un amour «qui ne change s'il rencontre en l'autre le changement» (Sonnet 116), se sont «transcendés vers l'autre», même si ce n'est pas au sens d'Emmanuel Lévinas ${ }^{184}$. Et l'on ne peut refuser une intuition de la transcendance absolue à l'auteur de l'Apologie quand il évoque le «Dieu caché » qui est seul « un realement estant» et qui, «par un seul maintenant emplit le tousjours » (II, 12, 588a). Mais cette évocation est empruntée à Plutarque ${ }^{185}$ et cette transcendance, contemplée par Montaigne de l'extérieur, semble même étrangère à son désir: «Ces humeurs transcendantes m'effrayent, comme les lieux hautains et

180 Sénèque, Questions naturelles, I, Préface ; cité par Montaigne : « O la vile chose... et abjecte que l'homme, s'il ne s'esleve au-dessus de l'humanité ! »

181 «To the Lady Margaret, Countesse of Cumberland», 1.98-99 (Poems and A Defence of Rhyme, éd. A. C. Sprague, Londres, Routledge \& Kegan, 1950).

182 Théologie Naturelle, éd. cit., $\mathrm{f}^{\circ} 5 \mathrm{v}^{\circ}$.

183 Ceci ne signifie point que Montaigne ou Shakespeare accepteraient l'idée que l'homme peut aussi «se transcender vers l'animalité » comme le suggère Grady, op. cit., p. 240-41.

${ }^{184}$ Pour Lévinas «la distance entre le même et l'autre est l'abîme même de la transcendance» et le sujet se transcende quand «il va vers l'Autre»: Fabio Cimarelli, Transcendance et éthique. Essai sur Lévinas, Bruxelles, OUSIA, 1989, p. 59, 207.

185 Aux chapitres 18 et 19 du dialogue Sur le E de Delphes. Mais Montaigne a pu se souvenir aussi de Sebond : «Dieu est une mer, un gouffre, \& un profond abysme d'essence, sans fons, sans bord, \& sans mesure» (Théologie Naturelle, chap. 12, $\mathrm{f}^{\circ} 18 \mathrm{r}^{\circ}$ ). 
inaccessibles » (III, 13, 1096c). L'être humain, paradoxalement, n'a selon l'Apologie «aucune communication à l'estre» (II, 12, 586a) entendu au sens métaphysique. Considérant la vie comme un instant «dans le cours infini d'une nuict eternelle (II, 12, 507b), et cependant luttant, d'une autre manière que Shakespeare, contre la tyrannie du temps, Montaigne cherche à prendre pleine possession du seul moment présent, à «l'estendre en pois» pour «arrester la promptitude de sa fuite par la promptitude de [sa] sesie» (III, 13, $1092 b)^{186}$.

L'intuition de la transcendance de l'Être me paraît absente chez Shakespeare, mais sur ce point je préfère laisser mon jugement en suspens. Je n'avancerai qu'une impression : ce qui s'est présenté avec le plus de force à son imagination, c'est l'idée que tout l'être sensible, tout le spectacle de l'univers, peu être amené à se dissoudre, «leave not a rack behind» (The Tempest, IV.i.151-58). L'idée que la vie pourrait être un songe était un lieu commun bien avant Calderon ; elle apparaît chez Montaigne, mais sans insistance ni émotion : «Ceux qui ont apparié nostre vie à un songe, ont eu de la raison, à l'avanture plus qu'ils ne pensoyent»(II, 12, 580b). Chez Shakespeare, en revanche, l'annonce d'une inévitable néantisation de l'être visible semble être le pressentiment vécu d'une transcendance en creux.

D'une transcendance en plein, Montaigne et Shakespeare ont eu néanmoins l'intuition à travers la fonction poétique du langage, car celle-ci peut procurer l'extase esthétique par laquelle «sujet et objet», dira Schopenhauer, «échappent au tourbillon du temps » ${ }^{187}$. C'est sur leur commune sensibilité à la poésie que je conclurai ces évocations parallèles. Montaigne, avouant une ambition littéraire qui lui ferait donner la préférence à l'enfantement d'un livre sur l'enfantement d'un enfant, écrivait: "je ne sçay si je n'aimerois pas mieux beaucoup en avoir produict ung, parfaictement bien formé, de l'acointance des muses, que de l'acointance de ma femme » (II, 8, 383b). Regretterait-il de ne pas écrire en vers? On pourrait le penser, car, « dès ma premiere

186 Selon Ulrich Langer l'auteur des Essais s'accorderait une "authoritative transcendence» à la manière du Créateur divin, mais son interprétation de sa déclaration liminaire «C'est icy un livre...» me paraît forcée; voir Divine and Poetic Freedom. Nom inalist Theology and Literature in France and Italy, Princeton U. P., 1990, p. 111.

${ }^{187}$ Voir John Keats. Poèmes, éd. R. Ellrodt, Paris, Imprimerie Nationale, 2000, p. 30. L'extase que ressent Florizel devant chaque action de Perdita s'y apparente: The Winter's Tale, IV.iv.136-46. 
enfance», dit-il, «la poësie a eu cela, de me transpercer et transporter» (I, 37, 228c). Mais la prose à ses yeux pouvait « reluire de la vigueur et hardiessse poétique », et c'est la quintessence de l'idéal humaniste de la Renaissance qu'il exprime en disant de la poésie, celle qu'il nomme « la bonne, l'excessive, la divine» (I, 37, 227a), que c'est la « vieille theologie», la «premiere philosophie» et «l'originel langage des Dieux » (III, 9, 974b).

Robert ELLRODT

Université Paris III - Sorbonne Nouvelle 